\title{
HIDROGEOMORFOLOGÍA EN ÁREAS TROPICALES: APLICACIÓN DEL ÍNDICE HIDROGEOMORFOLÓGICO (IHG) EN EL RÍO UTCUBAMBA (PERÚ)
}

\author{
HYDROGEOMORPHOLOGY ON TROPICAL AREAS: APPLICATION OF THE \\ HIDROGEOMORPHOLOGIC INDEX (IHG) IN THE UTCUBAMBA RIVER (PERU)
}

\author{
Elgar Barboza $^{1}$, Fernando Corroto $^{2}$, Rolando Salas $^{3}$, Oscar Gamarra $^{4}$, Daniel Ballarín ${ }^{5}$ y Alfredo Ollero
}

\begin{abstract}
Resumen
Los estudios hidrológicos y geomorfológicos se han incrementado en los últimos años indicando su importancia para entender el funcionamiento de los sistemas fluviales. En esta investigación se evaluó la calidad hidrogeomorfológica del río Utcubamba, ubicado en la cuenca del Amazonas, mediante la aplicación del Índice Hidrogeomorfológico (IHG) dividiéndolo en ocho sectores fluviales. Los resultados mostraron que el río Utcubamba está afectado por notables impactos antropogénicos que alteran las condiciones hidrogeomorfológicas, con un creciente deterioro de las mismas desde su nacimiento a su desembocadura. La calidad de las riberas es la más afectada en toda la cuenca, principalmente en el curso bajo debido a las explotaciones agropecuarias de carácter extensivo dominantes en este tramo. Por otra parte, el IHG presentó una adaptabilidad total a las condiciones del área de estudio, en un ámbito geográfico diferente y alejado de la Península Ibérica.

Palabras clave: Hidrología, IHG, índice, valoración, calidad, ríos, Utcubamba.
\end{abstract}

\begin{abstract}
Hydrological and geomorphological studies have increased in recent years indicating their importance to understand the functioning of river systems. In this research, the hydrogeomorphological quality of the Utcubamba River, located in the Amazon basin, was evaluated. By applying the Hydrogeomorphological Index (IHG) along its main watercourse the basin was divided into eight river sections. The results showed that the Utcubamba River is affected by significant anthropogenic impacts which modify the hydrogeomorphological conditions, with an increasing damage of them from its river source to his mouth. The quality of the riverbanks is most affected along the entire basin, mainly in the lower course due to extensive agriculture and livestock farms that are dominant in this part. Moreover, the IHG showed a complete adaptability to the conditions of the study area, in a different geographical area and far from the Iberian Peninsula.
\end{abstract}

Key words: Hydrology; IHG, index, quality, assessment, rivers, Utcubamba.

\section{Introducción.}

Los sistemas fluviales se caracterizan por un funcionamiento muy complejo con múltiples variables interactuantes a diferentes escalas espaciales $y$ temporales (Wheaton et al., 2011). Entre ellas, son especialmente relevantes los caudales líquidos y los caudales sólidos, que son las variables externas, que ejercen principalmente el control del sistema (Malavoi \& Bravard, 2010). Asimismo, influyen otras variables como el clima, los caracteres de cuenca, encajamiento, confinamiento, pendiente, materiales de fondo del valle y/o la propia vegetación (Ollero et al., 2011). En este contexto, se hacen trascendentales los componentes hidrológicos y geomorfológicos por el fuerte control que ejercen sobre el sistema fluvial en su conjunto (Horacio, 2015). La importancia de la hidrología en el funcionamiento del sistema fluvial se ve reflejada en el incremento, en los últimos años, del número de estudios relacionados a esta disciplina (Acreman \& Dunbar, 2004; Morote Seguido, 2014). Por su parte, la geomorfología juega un papel fundamental en los estudios hidrológicos debido a su influencia en las características y dinámica de una cuenca hidrográfica (Gil, 2012). Por tanto, hay que prestar máxima atención a las presiones humanas que generan impactos sobre estas variables hidrogeomorfológicas, pues el sistema fluvial se verá afectado por las mismas.

El deterioro de los ríos sigue en continuo aumento, en una línea de degradación ambiental a causa de las acciones humanas en el cauce y en cuencas que han alterado drásticamente muchas de las funciones hidrogeomorfológicas, ecológicas y bioclimáticas que, directa e indirectamente, influyen en los sistemas 
fluviales (Ibisate et al., 2011; Ollero et al., 2011). Las presiones e impactos son comunes en muchos ríos del planeta y muy frecuente en los ríos de Perú (García \& Otto, 2015), donde no hay estudios ni evaluaciones desde la perspectiva hidrogeomorfológica. En la cuenca del Utcubamba existen, sin embargo, estudios previos sobre la ecología de ecosistemas acuáticos, la calidad fisicoquímica y microbiológica (GarcíaHuamán et al., 2011), alertándose del mal estado de sus aguas a causa, principalmente, del vertido de residuos domésticos y agroquímicos (Chávez et al., 2016).

En la práctica, tanto la hidrología como la geomorfología fluvial ofrecen suficientes técnicas de análisis para evaluar la calidad hidrogeomorfológica con máximo detalle y fiabilidad; es decir, para valorar en qué estado de naturalidad se encuentran los ríos (Ollero et al., 2009).

En los últimos 20 años, en muchos países, especialmente en Europa, se han desarrollado metodologías de evaluación de la calidad hidrogeomorfológica a partir de la implantación de la Directiva Marco del Agua 2000/60/CE (DMA), que incluye los indicadores hidrogeomorfológicos en la evaluación de masas de agua superficiales; no obstante, los descriptores que propone la DMA dentro del estatus hidrogeomorfológico son muy pobres (Ollero et al., 2003), y en la práctica solo se emplean como apoyo de los descriptores biológicos para diferenciar entre el estado ecológico bueno y muy bueno. Entre las diferentes metodologías usadas, algunas se han centrado en la evaluación de alteraciones hidrológicas, como el Índice de Alteración Hidrológica en Ríos (IAHRIS) (SantaMaría \& Fernández Yuste, 2011), o en el transporte de sedimentos, como las Herramientas de Selección de Hidromodificación (Hydromodification Screening Tools (HST)) (Bledsoe et al., 2010). Otras caracterizan sistemas fluviales (Rosgen, 1996; Brierley \& Fryirs, 2000) o analizan hábitats, como la Encuesta de Hábitat de Río (River Habitat Survey (RHS)) (Raven et al., 1998) protocolo ampliamente aplicado en Gran Bretaña y otros países europeos; también ha sido utilizado el Índice de Funcionalidad Fluvial (IFF) (Siligardi et al., 2007) que, se ha aplicado en Europa y Chile (Dallafior et al., 2010). Un tercer grupo de metodologías establecen métricas y valoraciones numéricas con formato de índice, destacando el Índice de Calidad Morfológica (Morphological Quality Index (MQI)) (Rinaldi et al., 2013), que evalúa hasta 28 indicadores hidromorfológicos.

El Índice Hidrogeomorfológico (IHG, Ollero et al., 2008) se ha aplicado en diferentes estudios técnicos, propuestas de restauración fluvial, trabajos académicos y proyectos educativos (Ollero et al., 2009; Mora et al., 2012; Ballarín \& Rodríguez, 2013). Además, este índice ha sido bien valorado por su posible aplicación, de forma universal, a cualquier tipo de curso fluvial (Belletti et al., 2015). Así, el presente estudio constituye la primera aplicación sistemática completa del índice en ecosistemas tropicales, con los objetivos de diagnosticar la calidad hidrogeomorfológica del Utcubamba, testear el índice

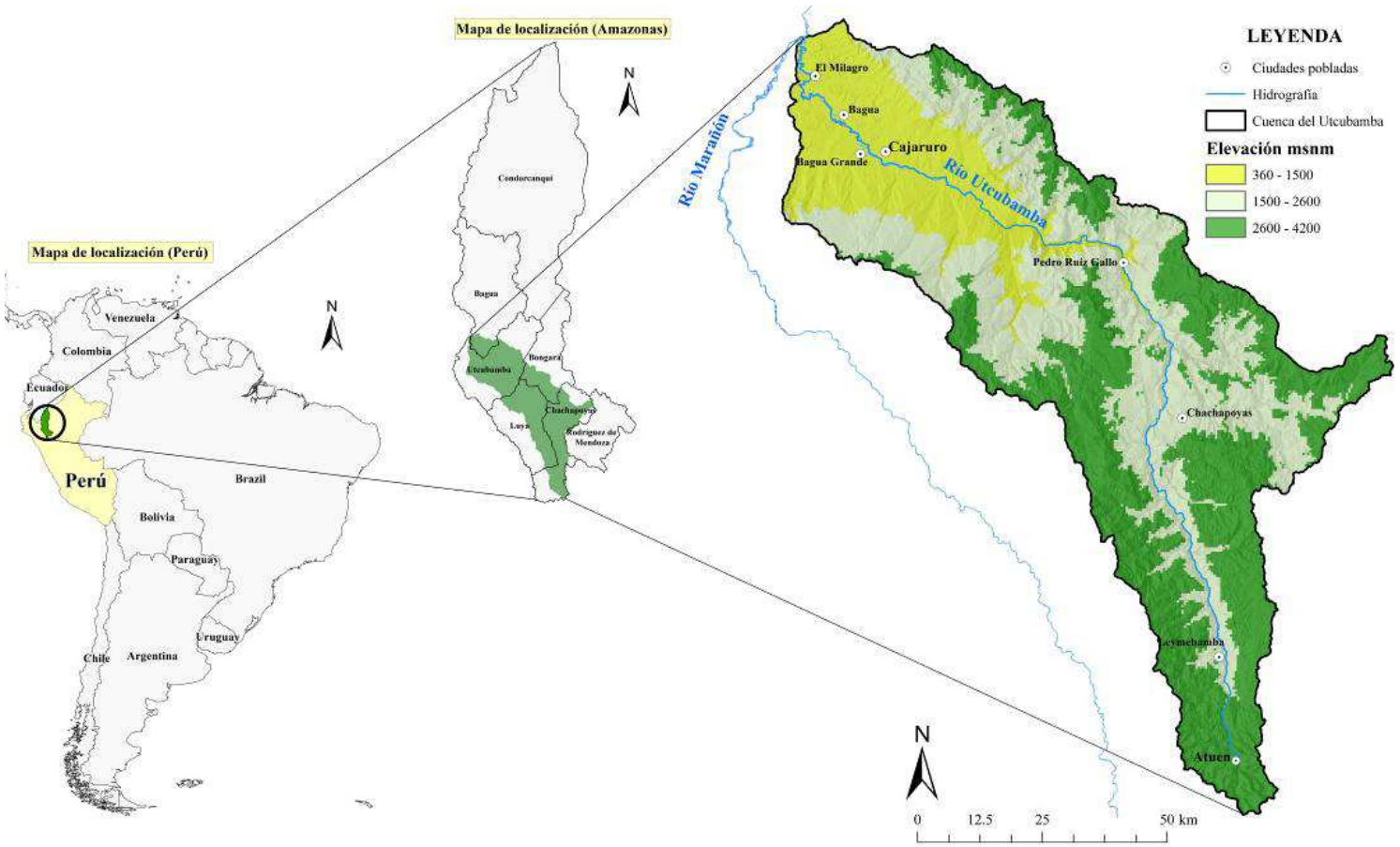

Figura 1. Localización del área de estudio en el departamento Amazonas (Perú), en el que se muestra los niveles altitudinales en la cuenca hidrográfica del río Utcubamba. 
IHG en un ámbito geográfico diferente de su creación y comprobar la eficacia del índice en el marco del proceso de simplificación del mismo.

\section{Materiales y métodos.}

Localización del área de estudio.

La cuenca del Utcubamba se ubica en el departamento de Amazonas (Perú), entre los $5^{\circ} 32^{\prime} 36^{\prime \prime}$ y $6^{\circ} 50^{\prime} 49^{\prime \prime}$ latitud sur, y $72^{\circ} 22^{\prime} 49^{\prime \prime}$ y $78^{\circ} 29^{\prime} 59^{\prime \prime}$ longitud oeste, con una superficie de $6650.07 \mathrm{~km}^{2}$ y una longitud de $250 \mathrm{~km}$ de cauce principal (Figura 1). Nace en el cerro Punta de Arena, cercano al caserío Atuen (provincia de Chachapoyas) y desemboca en el río Marañón (tributario del Amazonas).

La cuenca hidrográfica está englobada dentro de los ecosistemas de bosque tropical estacionalmente seco y bosque muy seco tropical, en la cuenca media y baja, y bosque pluvial montano en la cuenca alta. El bosque muy seco tropical, además, abarca los valles de los ríos Marañón, Chamaya y Chinchipe (LinaresPalomino, 2004). En cuanto a los bosques pluviales montanos, son aquellos localizados en la franja alta de la vertiente oriental y la faja entre los ríos Huancabamba y Chinchipe, entre 2500 y $3500 \mathrm{msnm}$ (León et al., 2006).

El relieve se caracteriza por presentar una altitud máxima de $4130 \mathrm{msnm}$ en la cabecera de la cuenca, en el cerro Los Chures, dentro de montañas de laderas muy empinadas a empinadas, y una mínima de 360 msnm que atraviesa en su recorrido colinas altas a bajas y valles en su desembocadura. Desde el punto de vista climático se caracteriza por una temperatura media anual de $29^{\circ} \mathrm{C}$ en la cuenca baja y $14^{\circ} \mathrm{C}$ en la cuenca alta. La pluviometría es escasa en la cuenca baja de $650 \mathrm{~mm} / a n ̃ o$, pero se incrementa a 1321 mm/año en la cuenca alta (Barboza et al., 2015). El caudal del río Utcubamba a la altura del Puente Caclic (cuenca media) es de $34.67 \mathrm{~m}^{3} / \mathrm{s}$, mientras que en la cuenca baja las dos estaciones de medición de caudal fueron en el Puente Corontachaca de $95.66 \mathrm{~m}^{3} / \mathrm{s}$ y en el sector más bajo, en el puente Milagro, que muestra un caudal de $211.24 \mathrm{~m}^{3} / \mathrm{s}$ (Maco, 2010).

La población concentrada a lo largo de la cuenca del Utcubamba es de 220048 habitantes, teniendo como núcleos principales a las ciudades de Leymebamba, Chachapoyas, Pedro Ruíz Gallo, Bagua Grande, Cajaruro, El Milagro y Bagua (INEI, 2015).

El uso del suelo predominante en la cuenca baja se enfoca en la agricultura de predominio arrocero, cafetalero y cultivos andinos, que representa el 53.03 \% del área total de la cuenca. La ganadería extensiva se desarrolla en un $15.51 \%$ con mayor intensidad en la cuenca media y alta. Finalmente, el $31.46 \%$ son tierras destinadas para la protección y conservación ambiental, incluyendo el uso urbano y cuerpos de agua (Ramírez, 2010). Sin embargo, las actividades de extracción de agregados (gravas, arenas y rocas) para la construcción, se han incrementado a lo largo y ancho del cauce principal del río Utcubamba (Leiva et al., 2015).

Delimitación de sectores funcionales.

Antes de aplicar el IHG fue necesario dividir el sistema fluvial en sectores funcionales, de manera que el índice se obtuviese individualmente. Para ello se empleó la clasificación de cursos fluviales propuesta por Díaz \& Ollero (2005), basada en dos variables geomorfológicas: la pendiente del cauce y la geomorfología del valle (estilo fluvial); así, con la ayuda de bases cartográficas y ortofotos se identificaron los cinco tipos de cursos fluviales: valle abierto extenso $(A)$ curso de cauce amplio que divagan sobre una llanura de inundación extensa, valle abierto de fondo encajado $(E)$, donde el cauce no puede divagar lateralmente y suele encajarse en terrazas con llanura de inundación reducida, valle encajado de fondo cóncavo (V), dentro del cual el cauce queda constreñido por las dos laderas de elevada pendiente y corredor ribereño estrecho, valle encajado de fondo plano (U), en el que el cauce puede divagar lateralmente pero queda limitado por las paredes escarpadas del valle y valle cerrado en cañón $(C)$, con un cauce muy estrecho, que carece de llanura de inundación y su corredor ribereño. Paralelamente se calculó la pendiente siguiendo la metodología propuesta por Horacio \& Ollero (2011). De esta manera, la estimación de la pendiente se realizó mediante software SIG a través de la combinación de un MDE y elementos vectoriales (líneas y puntos). Finalmente, y en función de los cinco tipos de cursos y la pendiente del cauce, el río Utcubamba quedó dividido en ocho sectores fluviales (Figura 2).

Evaluación hidrogeomorfológica.

El IHG se estructura en tres apartados: (1) Calidad Funcional del Sistema Fluvial (CFSF), (2) Calidad del Cauce (CC), y (3) Calidad de las Riberas (CR). A su vez, cada apartado se analiza en tres aspectos relevantes del funcionamiento hidrogeomorfológico de un río. Para cada sub-apartado se puede asignar un máximo de 10 puntos, por lo que la puntuación de cada apartado de calidad puede alcanzar 30 puntos, con una puntuación final del índice que oscila entre 0 y 90 puntos. Se utilizó la siguiente horquilla de puntuaciones (Tabla 1), para cada uno de los tres apartados del índice y para éste en su conjunto. Su aplicación requirió un conocimiento previo notable del sistema fluvial (documentación, datos hidrológicos, cartografía e imágenes satelitales), así como recorridos

Tabla 1. Calidad resultante de puntuaciones

\begin{tabular}{clc}
\hline $\begin{array}{c}\text { Puntuación } \\
\text { por apartado }\end{array}$ & $\begin{array}{l}\text { Calidad } \\
\text { hidrogeomorfológica }\end{array}$ & $\begin{array}{c}\text { Puntuación } \\
\text { total IHG }\end{array}$ \\
\hline $0-6$ & Muy Mala & $0-20$ \\
$7-13$ & Deficiente & $21-41$ \\
$14-19$ & Moderada & $42-59$ \\
$20-24$ & Buena & $60-74$ \\
$25-30$ & Muy Buena & $75-90$ \\
\hline
\end{tabular}

Fuente: Modificado de Ollero et al. (2009). 
longitudinales de campo con observaciones a detalle de elementos antrópicos, procesos de sedimentos, vegetación y crecidas. Análisis estadístico.

Las puntuaciones obtenidas para cada sector funcional con relación a cada apartado del IHG, fueron sometidas a una prueba Chi Cuadrado para conocer la independencia entre el tipo de sectores funcionales y el valor máximo y mínimo. Además, se utilizó la prueba de One-Way ANOVA para ver la diferencia significativa entre puntuación total del IHG obtenido en cada sector funcional y la tipología fluvial, y así, conocer cuál de los tres apartados influyó de manera directa en la puntuación final del IHG. Los análisis se realizaron con el software Statistix v. 9.0.

\section{Resultados.}

Delimitación de los sectores funcionales en relación a la morfología del valle.

La metodología usada permitió obtener ocho sectores a largo del curso fluvial (Figura 2). Los cursos abiertos extensos (A) representaron el $28 \%$ del cauce total del río. A continuación, los cursos encajados de fondo cóncavo (V) constituyeron el 24\%. Seguidamente se situaron los cursos encajados de fondo plano (U) y los cursos cerrados en cañón (C), que compusieron el $20 \%$ y el $16 \%$ del total del cauce, respectivamente. Por último, los cursos abiertos de fondo encajado (E) representaron el 12\% del total. Perfil longitudinal del cauce.

Representa las diferentes elevaciones del fondo del arroyo desde el nacimiento hasta la desembocadura del río (Senisterra et al., 2014). En el curso alto, el río Utcubamba presentó una pendiente media de $21.34 \%$ y una ruptura de pendiente que fluctuó entre 3.33 y $33.80 \%$ en las cercanías de la localidad La Joya.

En el curso medio la pendiente promedio fue de $14.38 \%$ con dos rupturas de pendiente que fluctuó en 21.88 a $69.54 \%$ en la unión de la desembocadura del río Sonche y en la confluencia de la quebrada Cocahuayco que varía de 7.47 a $60.70 \%$. En el curso bajo la pendiente promedio fue de $6.04 \%$. La pendiente máxima a lo largo de todo el cauce fue de 69.54\% y la mínima fue de $0.01 \%$ (Figura 3).

Calidad por apartados y calidad hidrogeomorfológica (IHG).

Los resultados obtenidos se muestran en la Figura 4. La Calidad Funcional del Sistema Fluvial (CFSF) mostró una puntación máxima de 21 en el sector funcional 01 (SF-01) (Figura 4A), seguido de los sectores funcionales 02 y 06 (Figuras 4B y 4F), ambos con 19 puntos; la mínima puntuación se registró en los sectores funcionales 05 y 08 (Figuras 4E y 4H), ambos

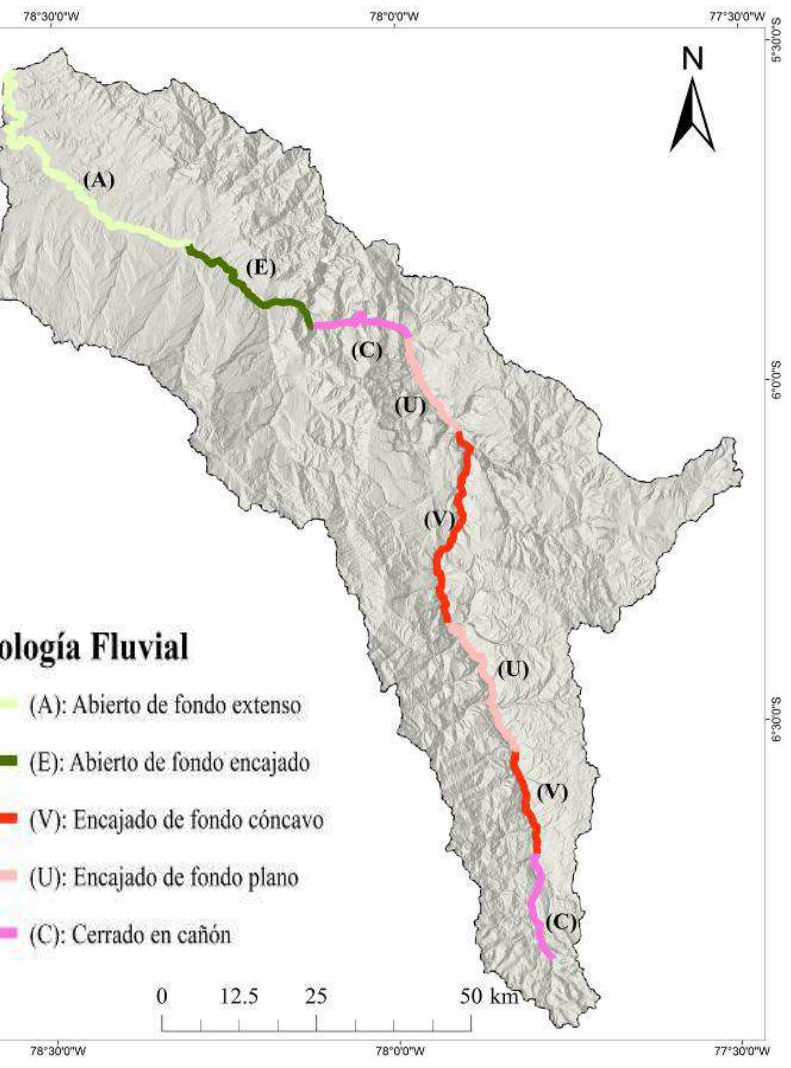

Figura 2. Tipología fluvial y sectores funcionales lasificados en el río Utcubamba.

con 14 puntos, seguidos del sector funcional 04 (Figura 4D) con 15 puntos. En la Calidad del Cauce (CC) las puntuaciones fueron desde 22, en el primer sector funcional del curso alto, a 14 en el último sector funcional del curso bajo. Finalmente, para la Calidad de Ribera (CR) las puntuaciones máximas se presentaron en los sectores funcionales 03 y 06 , con 15 y 16 puntos respectivamente (Figuras $4 \mathrm{C}$ y $4 \mathrm{~F}$ ). Por el contrario, la puntuación más baja se evidenció en el último sector funcional, con 7 puntos.

La valoración del IHG evidenció que la CFSF se encuentra entre los intervalos de buena a moderada, ubicándose la mejor calidad en el curso alto; de igual manera ocurre con la CC, con valores de calidad que van de buena (curso alto) a moderada (curso medio y bajo). Sin embargo, la CR presentó intervalos calidad deficiente (curso alto), moderada (curso medio) y deficiente (curso bajo). Se observa en líneas generales un deterioro de la calidad hidrogeomorfológica conforme se desciende en altitud a lo largo el curso fluvial, con siete sectores funcionales de calidad moderada y solo un sector con calidad deficiente (con 35 puntos).

Análisis estadístico.

La prueba de Chi Cuadrado muestra que la CFSF, CC y CR depende de la tipología fluvial de cada sector funcional $(p<0.001)$. La prueba de One-Way ANOVA reporta que no existen diferencias 


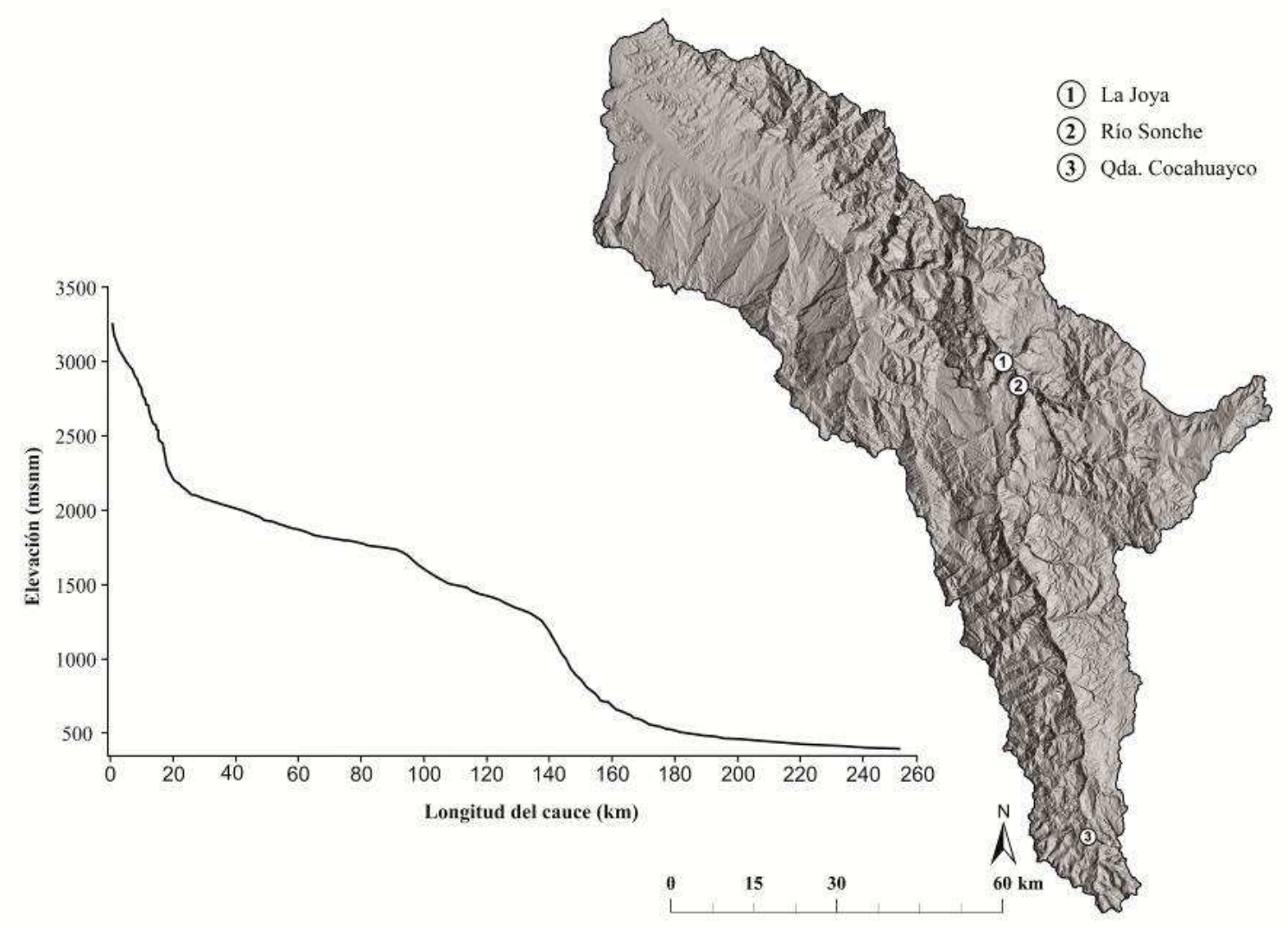

Figura 3. Perfil longitudinal del cauce principal del río Utcubamba y variaciones de la pendiente en el curso alto y medio del río.

estadísticamente significativas $(\mathrm{p}=0.0608)$ entre los valores del IHG y la tipología fluvial de cada sector funcional, cabe destacar que el curso A presenta la puntuación promedio más baja, con 10.667, valor por debajo del curso valor por debajo del curso abierto de fondo encajado (E), encajado de fondo plano (U) y cerrado en cañón $(\mathrm{C})$, que reportaron valores medios de $15.667,16.000$ y 16.500 respectivamente. Asimismo, los valores promedios más altos correspondieron con el curso de fondo cóncavo (V) con 17.500. A través de esta misma prueba, y al comparar de nuevo los valores obtenidos del IHG, en esta ocasión con los de CFSF, CC y CR, se evidencian diferencias altamente significativas $(\mathrm{p}=0.0027)$ entre los tres apartados, siendo la CR la más afectada, con una puntuación promedio de 13 , seguido de la CFSF con 17, quedando la $\mathrm{CC}$ con el promedio más alto con un valor de 18 .

\section{Discusión.}

Los resultados de la CFSF (buena a moderada) vienen derivados de actuaciones humanas (vertidos, urbanizaciones, represas y construcciones puntuales en las orillas cauce) que impactan directamente sobre el cauce (Ollero et al., 2009; García-Huamán et al., 2011). Los resultados de CFSF son relativamente similares a los reportados en otros estudios (Gustavson, et al., 2013; Villamarín et al., 2014) y, de acuerdo con estos, cuanto mayor fue la presencia de estas actuaciones la calidad funcional disminuyó.

La CC presenta intervalos mayores de calidad moderada, y calidad buena a deficiente; en este sentido, cabe destacar que el cauce es el elemento clave del sistema fluvial y construido por el propio río, el cual garantiza un sistema fluvial sano. Por tanto, esta calidad deficiente presente puede ser causada por la intervención antrópica a través de la construcción de infraestructuras como puentes, extracciones, desvíos, represas y canalizaciones, ocasionando la pérdida de naturalidad y geodiversidad, y poniendo en peligro la dinámica fluvial y el buen estado ecológico (Ollero et al., 2008; Ballarín \& Rodríguez, 2013).

La CR fue la que presentó los valores más bajos en relación a la CFSF y la CC. Las razones derivan de la explotación agrícola y ganadera existente sobre ellas a lo largo de toda la cuenca, lo que produce una fuerte degradación de la vegetación y de la biodiversidad que habita en las mismas (Buytaert et al., 2006). En este estudio, la conectividad longitudinal, la anchura del corredor ribereño y la estructura, naturalidad y conectividad transversal fueron los segmentos del bosque de ribera más modificados; estos resultados arrojaron valores muy bajos en este área del IHG, resultados similares a los obtenidos por Yalta et al. (2013) en su estudio realizado en la vegetación de ribera de afluentes de la cuenca media del río 
Utcubamba. La conservación de las zonas ribereñas es decisiva, ya que son hábitats cruciales para la supervivencia de las poblaciones, y constituyen un ambiente altamente productivo y regulador del intercambio de nutrientes entre ambiente terrestre $\mathrm{y}$ acuático (Méndez et al., 2015).

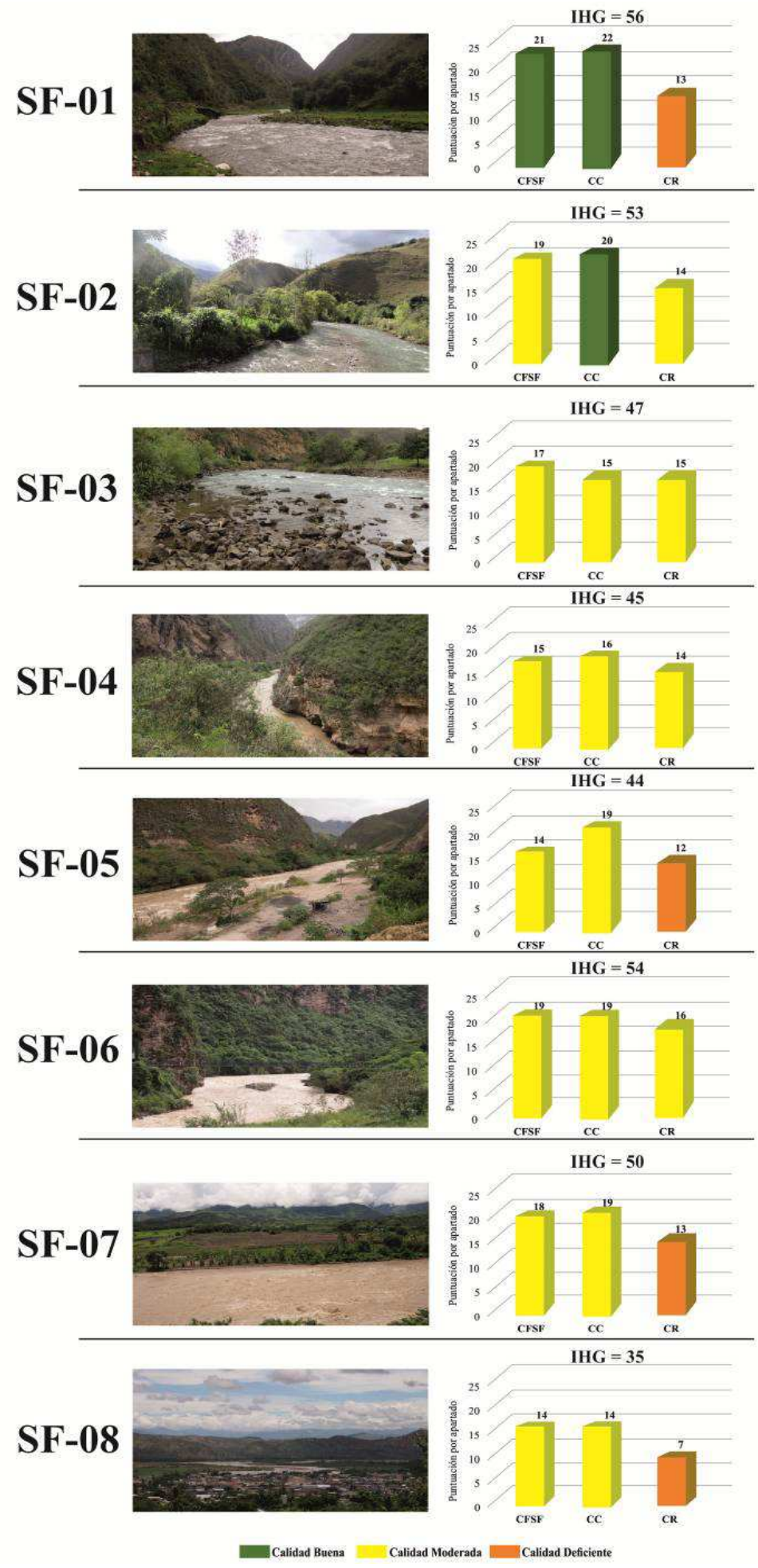

Figura 4. Imágenes de los sectores funcionales SF-01 (A), SF-02 (B), SF-03 (C), SF-04 (D), SF-05 (E), SF-06 (F), SF-07 (G), SF$08(\mathrm{H})$, e histogramas con las puntuaciones por apartado de cada sector (CFSF: Calidad Funcional del Sistema Fluvial; CC: Calidad del Cauce; CR: Calidad de Riberas).
En el curso alto, las causas que generan la degradación de las riberas derivan de la presión ganadera y agrícola, lo que genera discontinuidades longitudinales (Limachi, 2010). Estos cambios originados en curso superior influyen aguas abajo con un retardo temporal causa efecto, especialmente en los tramos medio e inferior de los cursos fluviales (Conesa \& Pérez, 2014), como se ve reflejado en el presente estudio.

En el curso medio se evidencia el impacto negativo de la carretera, paralela al cauce del río (Arévalo et al., 2008), junto al uso de suelo urbano. La morfología del valle en este curso medio es propicia a las expansiones urbanísticas de núcleos urbanos, por lo que estos tienden a ocupar zonas más próximas al cauce (Domenech et al., 2008). Otros factores influyentes en este curso medio son la extracción de agregados para las construcciones; estos aprovechamientos de minerales no metálicos han alterado profundamente aguas abajo el balance de agua y sedimento y, con ello, la morfología de los cauces. Asimismo, en la última década, se han incrementado a lo largo y ancho del cauce principal, trayendo consigo la reducción de orillas y riberas.

En el curso bajo la intervención antrópica es aún mayor, con extracciones agregados al igual que en el curso medio y extracción de agua por bombeo para uso agrícola. El bosque de ribera en este último sector se encuentra fuertemente destruido por la invasión de cultivo de arroz, lo que degenera en extensos valles compactados y salinizados a causa de la intensa actividad agrícola (Pérez-Castillo et al., 2013).

EL análisis estadístico reporta que no muestra independencia entre la calidad por apartado y la tipología fluvial de cada curso, en el caso de curso de valle extenso por lo general se tiene mayores impactos y por ende calidad hidrogeomorfológica más baja. Según Ibisate (2016) la calidad de cada sector funcional está vinculada a la presión sociodemográfica causada por la proliferación de usos artificiales en el cauce, la llanura de inundación y los 
cambios en los usos del suelo de la cuenca.

\section{Conclusiones.}

La calidad hidrogeomorfológica del río Utcubamba se sitúa en valores de moderada a deficiente, consecuencia de la presencia de acciones humanas (vertidos, urbanizaciones, presas y construcciones puntuales en las orillas del cauce) que impactan directamente sobre el sistema fluvial. En este sentido, es la calidad de las riberas a lo largo de toda la cuenca la que más se resiente por las actividades antrópicas.

El IHG ha sido aplicado sin ninguna modificación y ha mostrado una adaptación total a las condiciones del área de estudio en cada uno de los sectores funcionales evaluados, presentando resultados tan fiables en ecosistemas tropicales como los obtenidos en su lugar de origen.

\section{Agradecimientos.}

Esta investigación se realizó como parte del proyecto "Desarrollo de Índices Biológicos para la Evaluación Espacio - Temporal de la Calidad Ecológica del Agua en la Cuenca del Río Utcubamba, Región Amazonas, Perú"; financiado por el Programa Nacional de Innovación para la Competitividad y Productividad - FINCyT (Contrato $\mathrm{N}^{\circ}$ 221-FINCyTIA-2013) y ejecutado por el Instituto de Investigación para el Desarrollo Sustentable de Ceja de Selva INDES-CES de la UNTRM.

\section{Literatura citada.}

Acreman M. \& Dunbar M. J. 2004. Defining environmental river flow requirements - areview. Hydrology and Earth System Sciences. 8: 861-876.

Arévalo W., Guadalupe E., Pacheco R., Concepción L., Cabrera C. \& Iglesias S. 2008. Valoración de los paisajes naturales y del impacto paisajístico de las cataratas en la cuenca media del río Utcubamba. Revista del Instituto de Investigación de la Facultad de Ingeniería Geológica, Minera, Metalúrgica y Geográfica. 11: 68-75.

Ballarín D. \& Rodríguez I. 2013. Hidromorfología fluvial: algunos apuntes aplicados a la restauración de ríos en la cuenca del Duero. Confederación Hidrográfica del Duero. Valladolid, España.

Barboza E., Chichipe M. E. \& Salas R. 2015. Comparación de los MDE ASTER y SRTM en el análisis morfométrico de la cuenca del Utcubamba (Perú). INDES. 3: 86-98.

Belletti B. Rinaldi M. Buijse A. D., Gurnell A. M. \& Mosselman E. 2015. A review of assessment methods for river hydromorphology. Environmental Earth Sciences. 73: 2079-2100.

Bledsoe B. P., Hawley R. J., Stein D. E. \& Booth D. B. 2010. Hydromodification Screening Tools: Field manual for assessing channel susceptibility to Hydromodification. Technical Report 607, Southern California Coastal Water Research Project, Costa Mesa, California (USA).
Brierley G. J. \& Fryirs K. A. 2000. River styles, a geomorphic approach to catchment characterization: Implications for river rehabilitation in Bega catchment, New South Wales, Australia. Environmental Management. 25: 661-679.

Buytaert W., Celleri R., Debievre B., Cisneros F., Wyseure G., Deckers J. \& Hofstede R. 2006. Human impact on the hydrology of the Andean páramos. Earth-Science Reviews. 79: 53-72.

Chávez J., Leiva D. \& Corroto F. 2016. Caracterización fisicoquímica y microbiológica de las aguas residuales en la ciudad de Chachapoyas, Región Amazonas. Ciencia Amazónica. 6: 16-27.

Conesa C. \& Pérez P. 2014. Alteraciones geomorfológicas recientes en los sistemas fluviales mediterráneos de la Península Ibérica: Síntomas y problemas de incisión en los cauces. Revista de Geografía Norte Grande. 59: 2544.

Dallafior V., Canapel R., Burrows F. \& Siligardi M. 2010. IFFAR: Índice de funcionalidad fluvial en ríos andinos de la Región de La Araucanía. Eds. Sede Regional Villarrica, Pontificia Universidad Católica de Chile.

Díaz E. \& Ollero A. 2005. Metodología para la clasificación geomorfológica de los cursos fluviales de la cuenca del Ebro. Geographicalia. 44: 23-45.

Domenech S., Ollero A. \& Sánchez Fabre M. 2008. Núcleos de población en riesgo de inundación fluvial en Aragón: diagnóstico y evaluación para la ordenación del territorio. Geographicalia. 54: 17-44.

García E. \& Otto M. 2015. Caracterización ecohidrológica de humedales alto andinos usando imágenes de satélite multitemporales en la cabecera de cuenca del río Santa, Ancash, Perú. Ecología Aplicada. 14:115-125.

García-Huamán F. T., Torres-Delgado J. T. \& VergaraMedrano S. E. 2011. Calidad ecológica del agua del río Utcubamba en relación a parámetros fisicoquímicos y biológicos. Amazonas, Perú. SCIÉNDO. 14: 7-19.

Gil V. 2012. Cuenca alta del río Sauce Grande (Buenos Aires, Argentina). Cuaternario y Geomorfología. 1:133150.

Gustavson S. S. Cosme L. A. \& Trama F. A. 2013. Macroinvertebrados bentónicos como indicadores de la calidad de agua en la microcuenca San Alberto, Oxapampa, Perú. Apuntes de Ciencia y Sociedad. 3: 124-139.

Horacio J. \& Ollero A. 2011. Clasificación geomorfológica de cursos fluviales a partir de sistemas de información geográfica (S.I.G.). Boletín de la Asociación de Geógrafos Españoles 56: 373-396.

Horacio J. 2015. Medicina fluvial: Un nuevo paradigma en la conservación y restauración de ríos bajo el enfoque de la geomorfología. Ed. Jolube, Jaca.

Ibisate A., Ollero A., Sáenz de Olazagoitia A., Acín V., Granado D., Ballarín D., Herrero X., Horacio J. \& Mora D. 2016. Condiciones de referencia para la restauración de la geomorfología fluvial de los ríos de las cuencas de Oiartzun y Oria (Gipuzkoa). Cuaternario y Geomorfología. 30: 49-60.

INEI. 2015. PERÚ: Estimaciones y proyecciones de población por sexo, según departamento, provincia y distrito, 2000-2015. Instituto Nacional de Estadística e Informática. Lima.

Leiva Tafur D., Coronel E. \& Corroto F. 2015. Uso de diatomeas para medir el impacto de la extracción de 
agregados en ríos altoandinos, Amazonas, Perú. INDES 3: $33-42$.

León B., Pitman N., \& Roque J. 2006. Introducción a las plantas endémicas del Perú. Revista Peruana de Biología. 13: 9-22.

Limachi L. 2010. Socioeconomía, informe temático. Proyecto Zonificación Ecológica y Económica del departamento de Amazonas, convenio entre el IIAP y el Gobierno Regional de Amazonas. Iquitos.

Linares-Palomino R. 2004. Los bosques tropicales estacionalmente secos: I. El concepto de los bosques secos en el Perú. Arnoldoa. 11: 85-102.

Maco J. 2010. Hidrografía. Proyecto Zonificación Ecológica y Económica del departamento de Amazonas, convenio entre el IIAP y el Gobierno Regional de Amazonas. Iquitos.

Malavoi J. R. \& Bravard J. P. 2010. Eléments d'hydromorphologie fluviale appliquée. Ed. ONEMA. Francia.

Méndez W., Pacheco H., Cartaya S., Marcano A. \& León C. 2015. Caracterización hidroclimatológica y morfométrica de la cuenca del río San Julián (estado Vargas, Venezuela): aportes para la evaluación de la amenaza hidrogeomorfológica. Revista Colombiana de Geografía. 24: 133-156.

Mora D., Ballarín D., Montorio R., Zúñiga M., Ollero A., Durán C. \& Navarro P. 2012. Aplicación del índice hidrogeomorfológico IHG en el territorio aragonés de la cuenca del Ebro. Naturaleza aragonesa: Revista de la Sociedad de Amigos del Museo Paleontológico de la Universidad de Zaragoza. 28:35-42.

Morote Seguido A. F. 2014. La planificación y gestión de los recursos hídricos en España: aproximación a los principales grupos y líneas de investigación. Investigaciones Geográficas. 62: 113-125.

Ollero A. Ibisate A. González de Matauco V., Naverac A., Díaz E., Granado D. \& García J. H. 2011. Innovación y libertad fluvial. VII Congreso Ibérico sobre Gestión y Planificación del Agua "Ríos Ibéricos +10 . Mirando al futuro tras 10 años de DMA". Talavera de la Reina. 2011: 4-14.

Ollero A., Ballarín D. \& Mora D. 2009. Aplicación del índice hidrogeomorfológico IHG en la cuenca del Ebro. Guía metodológica. Confederación Hidrográfica del Ebro. Zaragoza.

Ollero A., Ballarín D., Díaz Bea E., Mora D., Sánchez Fabre M., Acín V., Echeverría M. T., Granado D., Ibisate L., Sánchez Gil L. \& Sánchez Gil N. 2008. IHG: Un índice para la valoración hidrogeomorfológica de sistemas fluviales. Limnetica. 27: 171-188.

Ollero A., Echeverría M. T., Sánchez Fabre M., Auría Izquierdo V., Ballarín D. \& Mora D. 2003. Metodología para la tipificación hidromorfológica de los cursos fluviales de Aragón en aplicación de la Directiva Marco de Aguas (2000/60/CE). Geographicalia. 44: 7-25.
Ollero A., Ibisate A., Horacio J., Ferrer C., Martín J. P., Acin V., Ballarin D., Díaz E., Granado D., Mora D. \& Sánchez M. 2011. Indicadores geomorfológicos para el seguimiento de la restauración fluvial. I Congreso Ibérico de Restauración Fluvial Restaurios. Leon. 346355.

Pérez-Castillo A. G.; Barboza-Mora R. \& Ramos-Matarrita J. F. 2013. Calidad del agua del refugio Mata Redonda y los arrozales colindantes, Guanacaste, Costa Rica. Agronomía Mesoamericana. 24: 379-392.

Ramírez J. 2010. Uso actual de la tierra. Informe temático. Proyecto Zonificación Ecológica y Económica del departamento de Amazonas, convenio entre el IIAP y el Gobierno Regional de Amazonas. Iquitos.

Raven P. J., Holmes N. T., Dawson F. H., Fox P. J., Everard M., Fozzard I. R. \& Rouen K. J. 1998. River Habitat Survey: the physical character or rivers and streams in the UK and Isle of Man. Environment Agency. Bristol.

Rinaldi M., Surian N., Comiti F. \& Bussettini M. 2013. A method for the assessment and analysis of the hydromorphological condition of Italian streams: the Morphological Quality Index (MQI). Geomorphology.180: 96-108.

Rosgen D. 1996. Applied River Morphology. Ed. Wildland Hydrology, Pagosa Springs, Colorado (USA).

Santa-María C. \& Fernández Yuste J. A. 2011. Obtención de escenarios de régimen ambiental de caudales (RAC) a partir del régimen natural: una nueva extensión del software IAHRIS. In: "I Congreso Ibérico de Restauración Fluvial - RestauraRíos". León, España.

Senisterra G. E., Rodríguez A. M., Gaspari F. J. \& Mazzucchelli M. G. A. 2014. Aspectos morfométricos de la cuenca alta del arroyo Napaleofú, provincia de Buenos Aires-Argentina. Revista Geográfica Venezolana. 2: 287-303.

Siligardi M., Avolio F., Baldaccini G., Bernabei S., Bucci M. S., Cappelletti C., Chierici E., Ciutti F., Floris B., Franceschini A., Mancini L., Minciardi M. R., Monauni C., Negri P., Pineschi G., Pozzi S., Rossi G., Sansoni G., Spaggiari R., Tamburro C. \& Zanetti M. 2007. Indice di funzionalità fluviale (IFF). Agenzia Nazionale per la Protezione dell'Ambiente e per i Servizi Tecnici. Roma.

Villamarín C., Prat N. \& Rieradevall M. 2014. Caracterización física, química e hidromorfológica de los ríos altoandinos tropicales de Ecuador y Perú. Latin American Journal of Aquatic Research.4 2: 1072-1086.

Wheaton J. M.; Gibbins C., Wainwright J., Larsen L. \& Mcelroy B. 2011. Preface: Multiscale Feedbacks in Ecogeomorphology. Geomorphology.126: 265-268.

Yalta J. R., Salas R. \& Alvarado L. 2013. Evaluación de la calidad ecológica del agua en las microcuencas de Chinata y Gocta, cuenca media del río Utcubamba, región Amazonas. Revista INDES: 1:14-28.

\footnotetext{
${ }^{1}$ Instituto de Investigación para el Desarrollo Sustentable de Ceja de Selva - Universidad Nacional Toribio Rodríguez de Mendoza, Calle Higos Urco 342, Chachapoyas-Perú, ebarboza@indes-ces.edu.pe.

${ }^{2}$ Instituto de Investigación para el Desarrollo Sustentable de Ceja de Selva - Universidad Nacional Toribio Rodríguez de Mendoza, Calle Higos Urco 342, Chachapoyas-Perú, fernando.corroto@untrm.edu.pe.

${ }^{3}$ Instituto de Investigación para el Desarrollo Sustentable de Ceja de Selva - Universidad Nacional Toribio Rodríguez de Mendoza, Calle Higos Urco 342, Chachapoyas-Perú, rsalas@indes-ces.edu.pe.
} 
${ }^{4}$ Instituto de Investigación para el Desarrollo Sustentable de Ceja de Selva - Universidad Nacional Toribio Rodríguez de Mendoza, Calle Higos Urco 342, Chachapoyas-Perú, ogamarra@indes-ces.edu.pe.

${ }^{5}$ Departamento de Geografía y Ordenación del Territorio - Universidad de Zaragoza, Calle Pedro Cerbuna, 12, 50009 Zaragoza (España), danielbf@unizar.es.

${ }^{6}$ Departamento de Geografía y Ordenación del Territorio - Universidad de Zaragoza, Calle Pedro Cerbuna, 12, 50009 Zaragoza (España), aollero@unizar.es. 